\title{
Model Predictions and Observed Performance of JWST's Cryogenic Position Metrology System
}

Sharon R. Lunt ${ }^{a}$, David Rhodes ${ }^{a}$, Andrew DiAntonio ${ }^{a}$, John Boland ${ }^{a}$, Conrad Wells ${ }^{a}$ Trevis Gigliotti ${ }^{b}$, Gary Johanning ${ }^{a}$ Harris Corp; ${ }^{b}$ Principal Point Geospatial Solutions; ${ }^{c}$ Geodetic Systems

INTRODUCTION

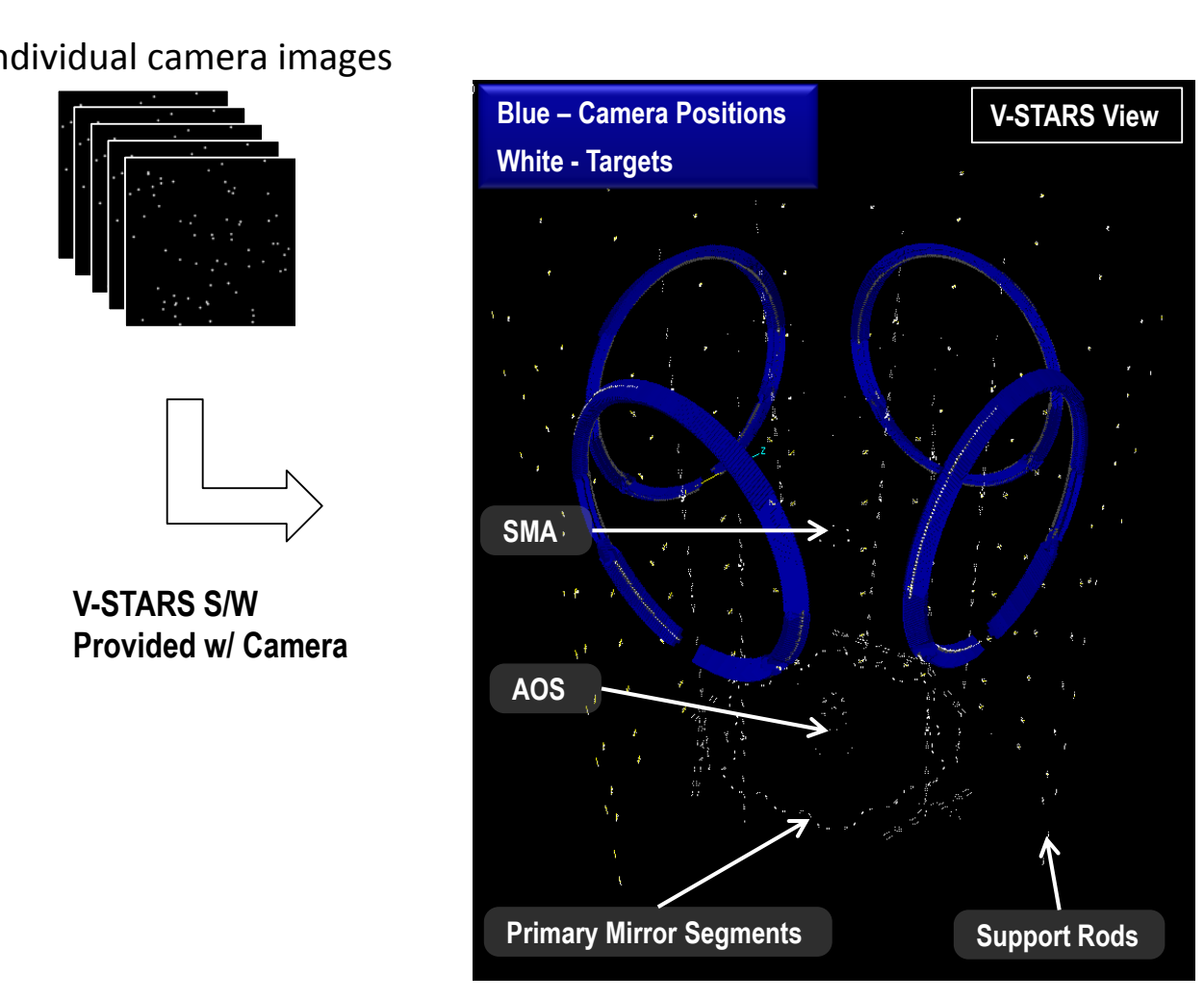

Photogrammetry Overview

PG cameras collect hundreds of

images

V-STARS software process images

("bundles") identifying individual

targets and determines target

locations \& uncertainties

-Locations defined for a specified

coordinate system

Data is exported

-Each target provided a unique repeatable identifier

Process data to Cardinal Data Report returns locations and uncertainties for optical assemblies

\section{CPM Overview}

For Thermal/Vacuum (T/V) tests of the JWST observatory, a set of four cameras on rotating windmill booms are used inside the helium shroud. The camera system records images of pecial targets placed on and about the OTE. The OTIS photogrammetric survey will be done primarily during conditions of a $30 \mathrm{~K}$ vacuum environment.

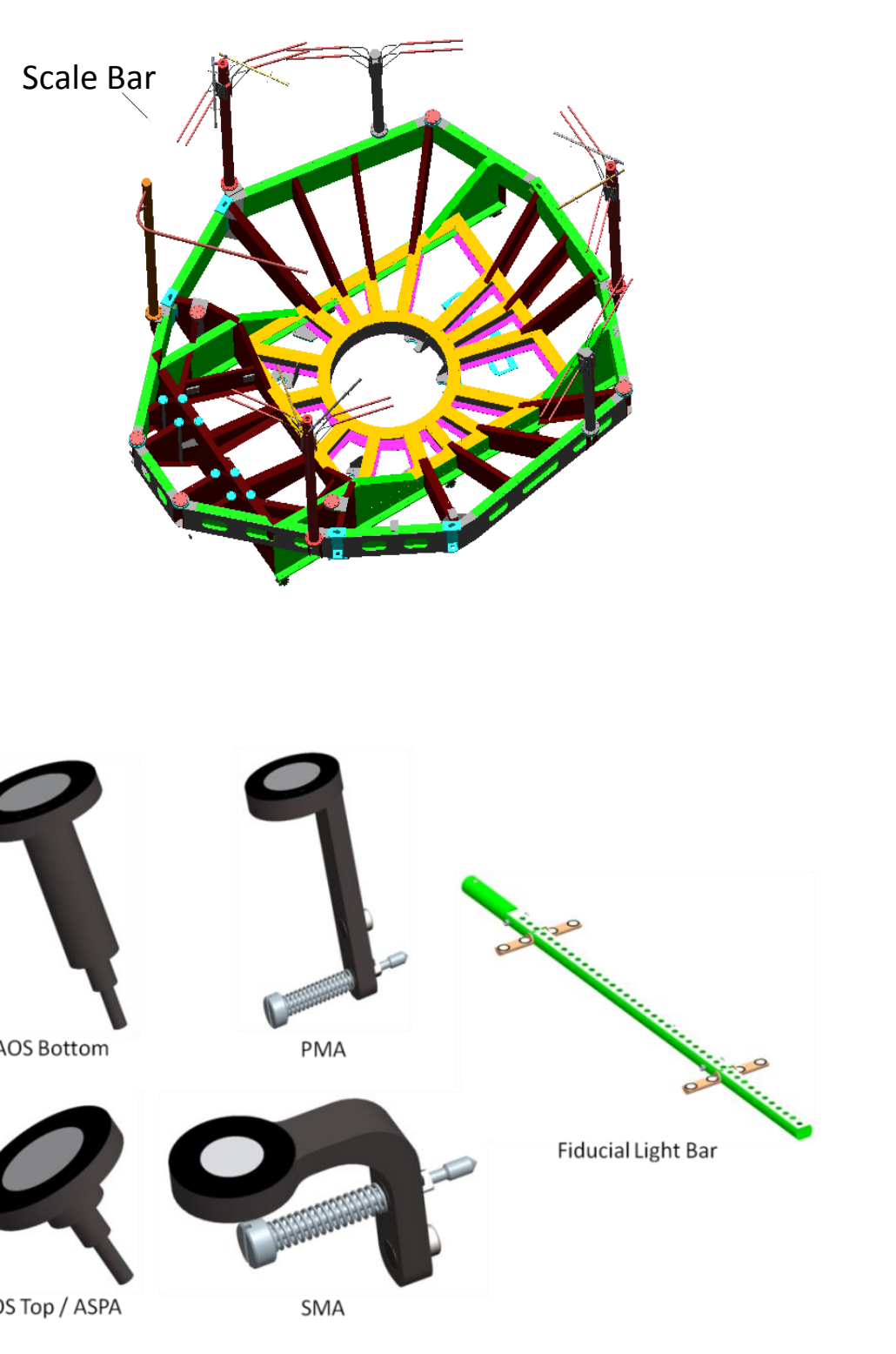

PG target components

Several types of targets are used in the chamber to ensure good

photogrammetric results.

- Scale bars - To scale a

photogrammetric measurement, at least one known distance must be present in the imagery.

- Target Assemblies - Individua reflective targets attached to hardware of interest.

- Code Targets - Code targets are a special type of target that the $\mathrm{V}$ STARS software can recognize to automatically calculate the position and orientation of the camera to aid bundle adjustment. Code targets are present on:

Chamber walls

Telescope rod sleeves

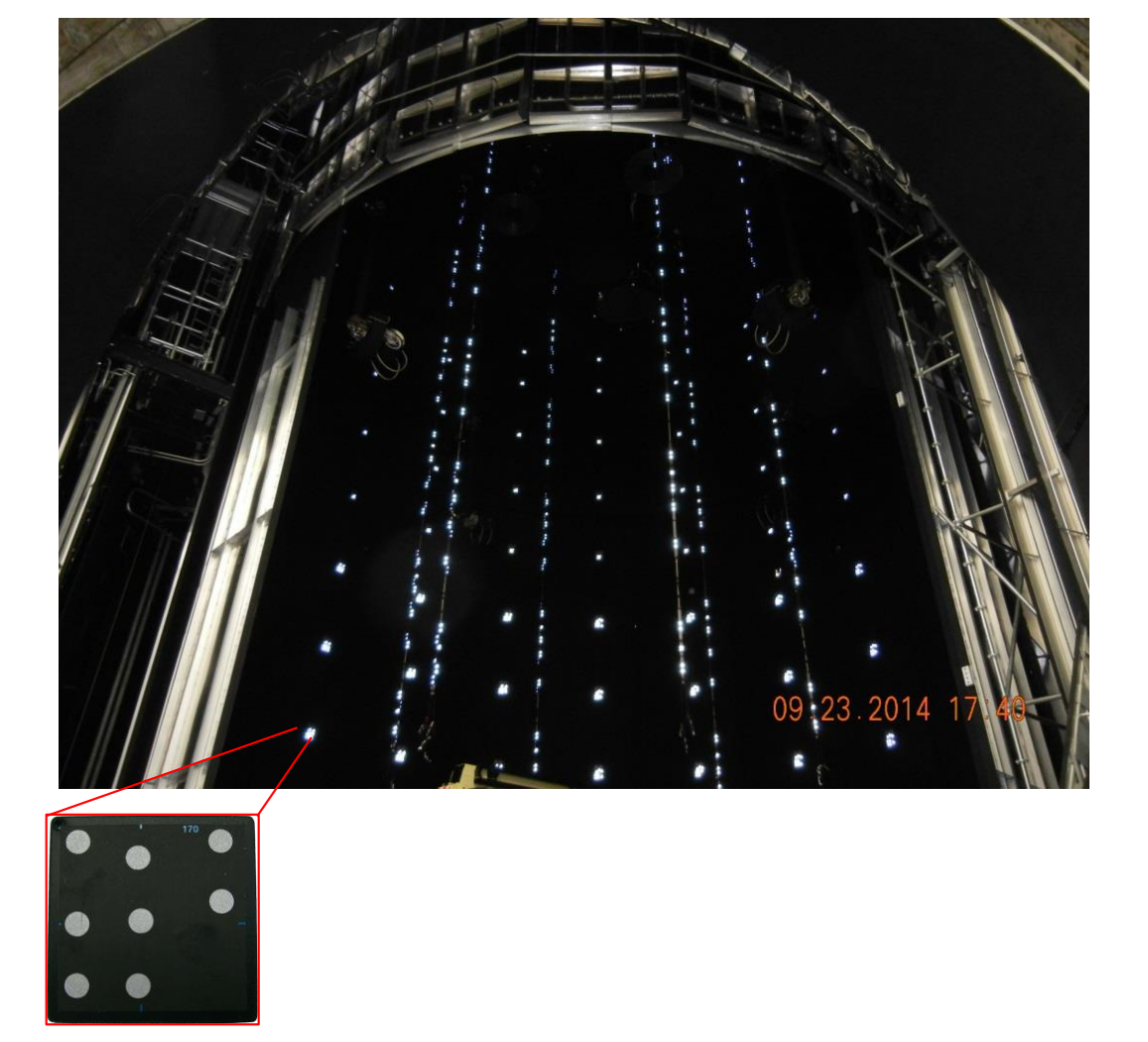

\section{COMPUTER MODEL CONSTRUCTION}

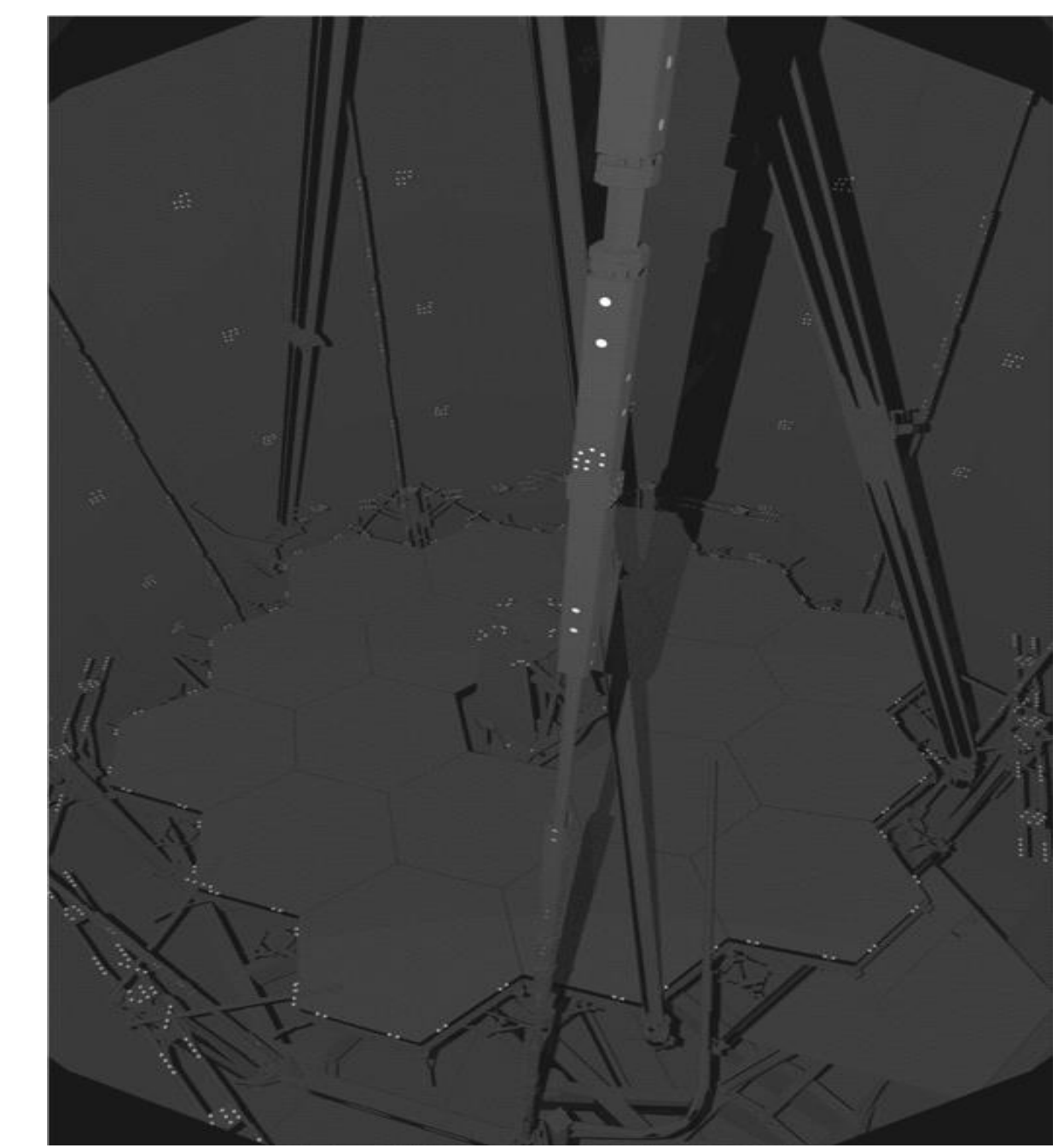

Possible QR

code for

video

(pending

approval)

The computer model was constructed using the Digital Image and Remote Sensing Image Generation (DIRSIG) version 4.5 software developed by Rochester Institute of Technology Digital Image and Remote Sensing Laboratory. DIRSIG performs end-to-end radiometric calculations from source to detector.

Model construction process:

- Extensive testing of actual targets at various sizes, angles, and distances was done with the INCA3 camera to determine distance and angle fall-off of target reflective material.

- CAD model of the OTIS configuration as well as the material properties of $3 \mathrm{M}$ retro reflective material used for the target material used to construct realistic model of the structures.

- Camera positioning and movement was incorporated into the definition of each modeled image.

- Image processing after the computer model generation was used to simulate sensor response and an adequate point spread function of the INCA camera system, and then converted to 8-bit.

The images were then imported into V-STARS for processing to determine the predicted PG measures of each point.

\section{RESULTS AND CONCLUSIONS}

The overall image residuals of the system were higher for the modeled imagery than actual imagery (1/13 of a pixel vs. $1 / 40$ of a pixel). The bundle adjustment and measurement uncertainty scale similarly to the image residuals between the modeled and actual results. The absolute accuracy was predicted well by the model.

\section{Conclusions}

- The close range photogrammetry system that is used in T/V testing of JWST hardware was modelled extensively prior to construction in order to verify performance, define camera pointing schemes, and assess effects of various proposed target configuration.

- A computer generated DIRSIG model predicted a higher level of image residuals and measurement uncertainty then observed in the actual system, but correctly predicted the error in absolute measurement. When the difference between model and actual image residuals are accounted for, the measurement uncertainty from the DIRSIG model is similar to that found in chamber T/V verification testing.

- The CPM system was predicted by the DIRSIG model to meet the requirements of the error budget and inchamber testing confirmed those predictions. The measurement uncertainty of the CPM is $<0.1 \mathrm{~mm}$, the absolute positional accuracy at the AOS and PM level is $<0.1 \mathrm{~mm}$, and the absolute positional accuracy is $<0.15 \mathrm{~mm}$ at the SM level.

- The CPM has been successfully used in 3 chamber tests and will be used in the final OTIS testing phase. CCT Configuration:

The hardware configuration used for CCT had 8 calibration 作 we system to measure the

\section{GSE1 Configurataion} PG targets attached by mounting assemblies to (he reference for
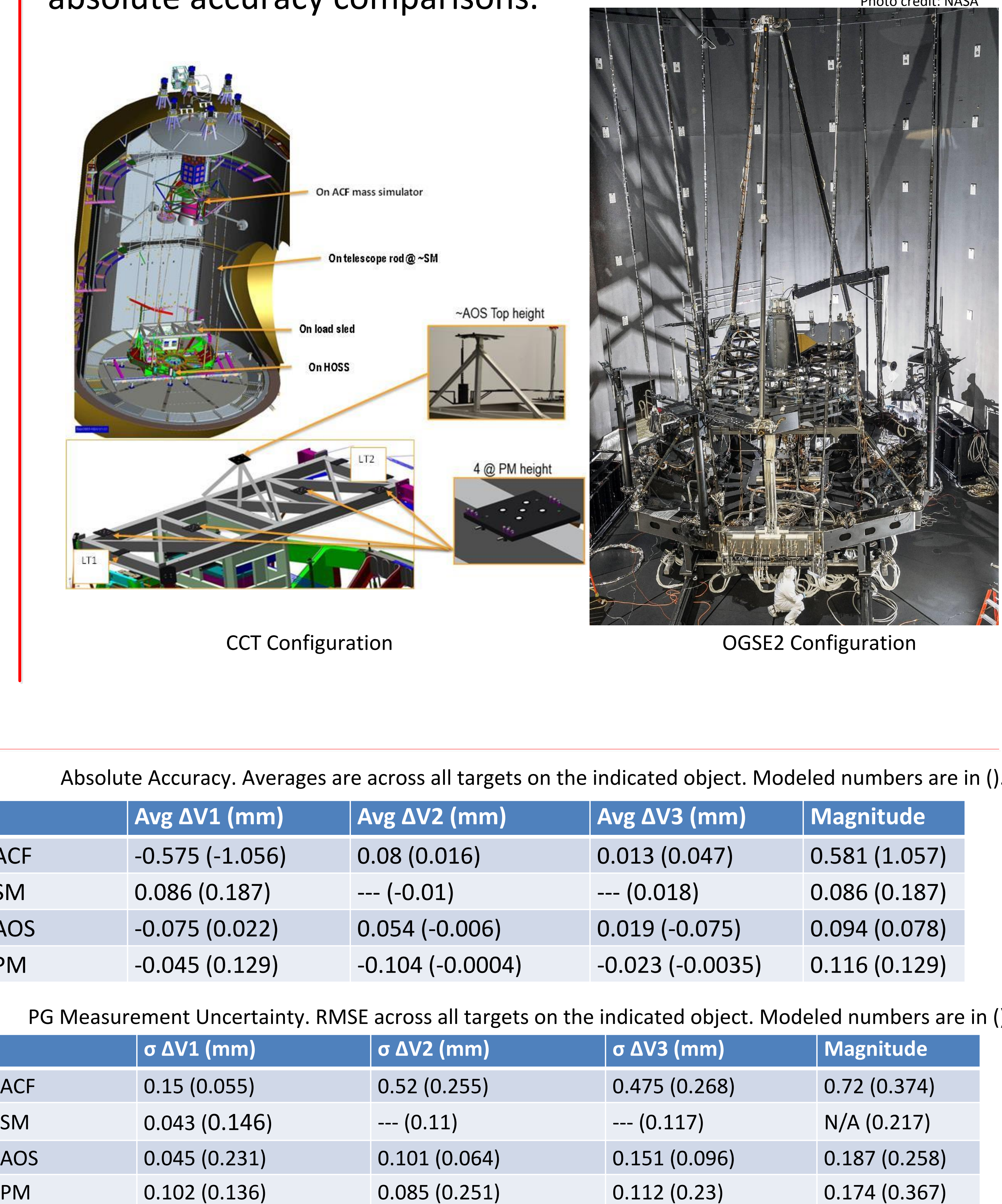
$\quad 0.174(0.367)$ 